\title{
Stability and production of positron-diatomic molecule complexes
}

\author{
Massimo Mella ${ }^{a}$ \\ Dipartimento di Chimica Fisica ed Elettrochimica, Universita' degli Studi di Milano, via Golgi 19, \\ 20133 Milano, Italy \\ Dario Bressanini ${ }^{\text {b) }}$ and Gabriele Morosi ${ }^{\mathrm{c})}$ \\ Dipartimento di Scienze Chimiche, Fisiche e Matematiche, Universita' dell'Insubria, via Lucini 3, \\ 22100 Como, Italy
}

(Received 9 April 2001; accepted 2 May 2001)

\begin{abstract}
The energies at geometries close to the equilibrium for the $e^{+} \mathrm{LiF}$ and $e^{+} \mathrm{BeO}$ ground states were computed by means of diffusion Monte Carlo simulations. These results allow us to predict the equilibrium geometries and the vibrational frequencies for these exotic systems, and to discuss their stability with respect to the various dissociation channels. Since the adiabatic positron affinities were found to be smaller than the dissociation energies for both complexes, we propose these two molecules as possible candidates in the challenge to produce and detect stable positron-molecule systems. Moreover, low-energy positron scattering on $\mathrm{LiF}$ and $\mathrm{BeO}$ targets may show vibrational Feshbach resonances as fingerprints of the existence of stable ground states of $e^{+} \mathrm{LiF}$ and $e^{+} \mathrm{BeO}$.

(C) 2001 American Institute of Physics. [DOI: 10.1063/1.1381009]
\end{abstract}

Despite the wide diffusion of positron and positronium (Ps) based analytical techniques to study solids, ${ }^{1}$ polymers, ${ }^{2}$ solutions, ${ }^{3}$ and organic molecules in the gas phase, ${ }^{4,5}$ a direct observation of the compounds between the positron and an atom or a molecule is still lacking. In fact the $\Gamma_{2 \gamma}$ annihilation rate from positron annihilation lifetime spectroscopy and angular correlation annihilation radiation are the only standard measurements carried out during the interaction positron-matter. The prediction of these observable is required to infer the formation of the positronic compounds, a task that appears complex, especially for heavy atoms and ions or large molecules, due to the high accuracy that is needed for the wave function that describes the complexes.

The theoretical work on positron-containing systems is scarce, and probably this is due to the difficulty in describing accurately the electron-positron correlation using standard quantum chemistry methods like self-consistent field, configuration interaction, and coupled cluster methods. ${ }^{6}$

Two more approaches have been pursued during the last few years, namely density functional theory $(\mathrm{DFT})^{7}$ and variational calculations based on explicitly correlated Gaussian (ECG) trial wave functions. ${ }^{8,9}$ They also suffer from practical drawbacks. Although DFT methods have a convenient scaling of the computational cost versus the system complexity, the exact exchange-correlation potential between electrons and the correlation potential between electrons and positron are only approximately known. As far as ECG wave functions are concerned, two groups ${ }^{8,9}$ showed that accurate results can be obtained even for positroncontaining systems. Unfortunately, the ECG wave functions suffer from the fast increase of the computational cost with the number of particles, therefore preventing their use for

\footnotetext{
${ }^{a)}$ Electronic mail: Massimo.Mella@unimi.it

b)Electronic mail: Dario.Bressanini@uninsubria.it

${ }^{c)}$ Electronic mail: Gabriele.Morosi@uninsubria.it
}

medium and large systems. Nevertheless, accurate results can be obtained by employing the frozen-core approximation for atoms and molecules. ${ }^{8}$

In our ongoing project to study positronic compounds as a way to understand matter-antimatter interactions and to predict the existence of a bound state for positron-atom or positron-molecule complexes, ${ }^{10-15}$ we employ the fixed node diffusion Monte Carlo (FN-DMC) method. ${ }^{16}$ This technique is known to be able to recover most of the correlation energy between electrons and between electrons and a positron. ${ }^{10-15,17-19}$ Although FN-DMC is a powerful technique, it is not easy to reduce the nodal error introduced by the fixed node approximation. This result might be achieved in principle by employing more accurate trial wave functions or resorting to the nodal release technique, but both approaches do not easily apply to large systems, i.e., more than ten electrons, due to their computational cost. Nevertheless, the FN-DMC method has given accurate positron affinities, as well as electron affinities, ${ }^{20}$ for systems up to twelve electrons, both atoms and molecules, exploiting the cancellation of nodal errors. ${ }^{12}$

In the quest for stable positronic complexes, we studied the potential surface for $e^{+} \mathrm{LiH}$ by FN-DMC calculations ${ }^{21}$ and found that the equilibrium distance and the vibrational transitions are different from those of $\mathrm{LiH}$, opening the possibility for a spectroscopic detection of this compound. However, the $\mathrm{LiH}$ adiabatic positron affinity (APA) is larger than the dissociation energy (DE), and a third body would be required to dissipate the excess energy. We suggested to start from a van der Waals complex of $\mathrm{LiH}$ with a rare gas, and to attach the positron to this so that the rare gas should dissipate the excess energy. Similar consideration can be extracted from the work of Mitroy and Ryzhikh, ${ }^{22}$ where they employed a full nonadiabatic approach and ECG functions to establish the stability of $e^{+} \mathrm{LiH}$.

In this work, we investigate other systems to see if we 
TABLE I. Total energy at various internuclear distances. All values are in atomic units.

\begin{tabular}{ccc}
\hline \hline & $R$ & \multicolumn{1}{c}{$\langle E\rangle$} \\
\hline$e^{+} \mathrm{LiF}$ & 2.955 & $-107.4243(8)$ \\
& 3.200 & $-107.4291(8)$ \\
& 3.400 & $-107.4249(10)$ \\
$e^{+} \mathrm{BeO}$ & 3.500 & $-107.4176(8)$ \\
& 2.30 & $-89.7975(13)$ \\
& 2.40 & $-89.8089(15)$ \\
& 2.51 & $-89.8108(18)$ \\
& 2.75 & $-89.7998(14)$ \\
\hline \hline
\end{tabular}

can find a molecule whose APA is smaller than the DE. This might allow the positron to remain temporarily attached to the molecule in a metastable state, or to increase the chances of forming the positron complex with the intervention of a third body. If the spectroscopic properties of the positronic compound differ from those of the parent molecule, it could be a good candidate for experimental observation.

We have performed accurate calculations of the total energy for $e^{+} \mathrm{BeO}$ and $e^{+} \mathrm{LiF}$ systems at various internuclear distances by means of FN-DMC. These results allow us to obtain the equilibrium distances for both molecules and to compute the vibrational frequencies.

In the FN-DMC algorithm we sample a distribution of configurations in $3 N$ dimensional space that represents $\Psi_{0} \Psi_{T}$, where $\Psi_{0}$ is the ground state wave function having the same nodal surfaces as the trial wave function $\Psi_{T}$. Using this distribution we obtain a MC estimate of the fixed node energy $E_{0}$ using the mixed estimator

$$
E_{0}=\frac{1}{N} \sum_{i=1}^{N} E_{\text {elec }}\left(\mathbf{R}_{i}\right)=\frac{1}{N} \sum_{i=1}^{N} \frac{H \Psi_{T}\left(\mathbf{R}_{i}\right)}{\Psi_{T}\left(\mathbf{R}_{i}\right)} .
$$

In our calculations the trial wave function $\Psi_{T}$ is

$$
\Psi_{T}=\operatorname{Det}\left|\phi_{\alpha}\right| \operatorname{Det}\left|\phi_{B}\right| e^{U\left(r_{\mu \nu}\right)} \Omega\left(\mathbf{r}_{p}, r_{p \nu}\right),
$$

where $\phi_{\alpha, \beta}$ are orbitals and $e^{U\left(r_{\mu \nu}\right)}$ is the electronic correlation factor used by Schmidt and Moskowitz in their works on atoms and ions. ${ }^{23,24}$ We refer to our previous works ${ }^{11-13}$ for the complete form of our trial wave functions and the details of the optimization procedure..$^{25,26}$

All the FN-DMC simulations were carried out using a target population of 5000 configurations and a time step of 0.001 hartee $^{-1}$. A few more simulations employing a time step of 0.0005 hartree $^{-1}$ were run to check for the absence of the time step bias in the mean energy values. The FN-DMC energy results for various internuclear distances of $e^{+} \mathrm{LiF}$ and $e^{+} \mathrm{BeO}$ are shown in Table $\mathrm{I}$.

We fitted these energy values by means of a secondorder polynomial and computed equilibrium geometrical parameters and the fundamental vibrational wave number $\omega_{e}$ for the two complexes $e^{+7} \mathrm{Li}^{19} \mathrm{~F}$ and $e^{+9} \mathrm{Be}^{16} \mathrm{O}$. All the results are collected in Table II.

Comparing our results with the experimental values, ${ }^{27}$ also in Table II, for ${ }^{7} \mathrm{Li}^{19} \mathrm{~F}$ and ${ }^{9} \mathrm{Be}^{16} \mathrm{O}$, we note that after the addition of the positron both molecules have larger equilibrium distances and vibrational wave numbers. While the in-
TABLE II. Equilibrium properties for $e^{+7} \mathrm{Li}^{19} \mathrm{~F}, e^{+9} \mathrm{Be}^{16} \mathrm{O},{ }^{7} \mathrm{Li}^{19} \mathrm{~F}$, and ${ }^{9} \mathrm{Be}^{16} \mathrm{O}$. Adiabatic (APA) and vertical (VPA) positron affinity of $\mathrm{LiF}$ and $\mathrm{BeO}$. Energies for the two lowest dissociation thresholds $e^{+} \mathrm{M}+\mathrm{X}$, and $\mathrm{PsX}+\mathrm{M}^{+}$, and dissociation energy (DE) for $e^{+} \mathrm{LiF}$ and $e^{+} \mathrm{BeO}$. All quantities are in atomic units.

\begin{tabular}{lcccc}
\hline \hline & $e^{+7} \mathrm{Li}^{19} \mathrm{~F}$ & $e+{ }^{+9} \mathrm{Be}^{16} \mathrm{O}$ & ${ }^{7} \mathrm{Li}^{19} \mathrm{~F}$ & ${ }^{9} \mathrm{Be}^{16} \mathrm{O}$ \\
\hline$E_{\min }$ & $-107.4290(10)$ & $-89.8108(16)$ & $-107.4069(9)$ & $-89.7854(13)$ \\
$R_{e}$ & 3.18 & 2.53 & $2.955^{\mathrm{a}}$ & $2.515^{\mathrm{a}}$ \\
$\omega_{e}\left(\mathrm{~cm}^{-1}\right)$ & 1073 & 1537 & $910.34^{\mathrm{a}}$ & $1487.52^{\mathrm{a}}$ \\
$R_{0}$ & 3.20 & 2.55 & & \\
$\mathrm{APA}$ & & & $0.022(1)$ & $0.025(2)$ \\
$\mathrm{VPA}$ & & & $0.017(1)$ & $0.025(2)$ \\
$\mathrm{DE}$ & $0.077(1)$ & $0.090(2)$ & & \\
$e^{+} \mathrm{M}+\mathrm{X}$ & $-107.2499(3)$ & $-89.7208(4)$ & & \\
$\mathrm{PsX}+\mathrm{M}^{+}$ & $-107.3518(8)$ & $-89.6425(5)$ & & \\
\hline \hline
\end{tabular}

${ }^{\mathrm{a}}$ Reference 27.

crease of $R_{e}$ is similar to the one we found for $e^{+} \mathrm{LiH}$ (Ref. $21)$ and can be rationalized by invoking the repulsive interaction of the positron with the nuclei, the increase of stiffness of the two bonds is an unexpected result. However, it must be pointed out that the computed wave numbers have an estimated statistical accuracy of the order of $10 \%$, and this means that care must be taken in discussing the change of this property.

In a previous work ${ }^{12}$ we computed the total energies for $\mathrm{LiF}[-107.4068(9)$ hartree $]$ and $\mathrm{BeO}[-89.7854(13)$ hartree] at their equilibrium distances by means of FN-DMC. Together with the $E_{\min }$ values for the positron systems shown in Table II, these energies allow us to compute the adiabatic positron affinity (APA) for these two systems, namely 0.022(1) hartree for $e^{+} \mathrm{LiF}$, and 0.025(2) hartree for $e^{+} \mathrm{BeO}$. These two values are smaller than the APA for the $e^{+} \mathrm{LiH}[0.0366(1)$ hartree]. This result was already observed for the vertical PA, ${ }^{12}$ and is in contrast with the fact that the dipole moments of $\operatorname{LiF}(\mu=6.33 \mathrm{D})$ and $\mathrm{BeO}(\mu=6.26 \mathrm{D})$ are larger than the one of $\operatorname{LiH}(\mu=5.88 \mathrm{D}){ }^{28}$ This indicates that the dipole moment is not sufficient to predict a qualitative trend in the PA, and that this value strongly depends on the specific features of each molecule.

It is interesting to notice that the three stable complexes $e^{+} \mathrm{LiH}, e^{+} \mathrm{LiF}$, and $e^{+} \mathrm{BeO}$ give the two stable systems $e^{+} \mathrm{Be}$ and $e^{+} \mathrm{Mg}^{8}$ in the united atom limit. Conversely, the unbound ${ }^{12} e^{+} \mathrm{HF}$ and $e^{+} \mathrm{H}_{2} \mathrm{O}$ give in this limit $e^{+} \mathrm{Ne}$ that is also not bound. These facts seem to suggest a connection between the (un)stability of the positronic system in the united atoms limit and the (un)stability of a positrondiatomic molecule complex. More specifically, one might infer that if the complex $e^{+} \mathrm{A}$ in the united atom limit is stable, than the complex $e^{+} \mathrm{MX}$ coming from it is also stable. For example, recalling that $e^{+} \mathrm{Mg}, e^{+} \mathrm{Ca},{ }^{29} e^{+} \mathrm{Zn},{ }^{30}$ and $e^{+} \mathrm{Cd}^{31}$ are bound, it is easy to predict that $e^{+} \mathrm{NaH}, e^{+} \mathrm{KH}$, and $e^{+} \mathrm{LiCl}$ should be bound due to the strong dipolar moment of the parent molecule. The systems $e^{+} \mathrm{BeS}, e^{+} \mathrm{CuH}$, $e^{+} \mathrm{AgH}, e^{+} \mathrm{NeHe}$, and $e^{+} \mathrm{ArHe}$ are more intriguing as these are only slightly polar. Since $e^{+} \mathrm{Be},{ }^{8} e^{+} \mathrm{Cu},{ }^{32}$ and $e^{+} \mathrm{Ag}^{33}$ are bound, there are chances that also $e^{+} \mathrm{BeS}, e^{+} \mathrm{CuH}$, and $e^{+} \mathrm{AgH}$ might be stable. Instead, neither $\mathrm{Ne}$ nor $\mathrm{Ar}$ are ex- 
TABLE III. Energy (in hartree) of the possible dissociation fragments for $e^{+} \mathrm{LiF}$ and $e^{+} \mathrm{BeO}$.

\begin{tabular}{cc}
\hline \hline & $\langle E\rangle$ \\
\hline $\mathrm{Li}^{+}$ & $-7.279913^{\mathrm{a}}$ \\
$e^{+} \mathrm{Li}$ & $-7.532323^{\mathrm{a}}$ \\
$e^{+} \mathrm{Be}$ & $-14.669042^{\mathrm{a}, \mathrm{b}}$ \\
$\mathrm{Be}$ & $-14.667355^{\mathrm{a}}$ \\
$\mathrm{O}$ & $-75.0518(4)^{\mathrm{c}}$ \\
$\mathrm{F}$ & $-99.7176(3)^{\mathrm{c}}$ \\
$\mathrm{PsO}$ & $-75.3177(5)^{\mathrm{d}}$ \\
$\mathrm{PsF}$ & $-100.0719(8)^{\mathrm{d}}$ \\
\hline \hline${ }^{2}$ Reference 8. & ${ }^{\mathrm{c}}$ Reference 36 \\
${ }^{\mathrm{b}}$ Reference 34. & ${ }^{\mathrm{d}}$ Reference 11.
\end{tabular}

pected to bind a positron, so that binding, if any, in $e^{+} \mathrm{NeHe}$ and $e^{+}$ArHe could be due to the superposition of polarization effects of the couple of atoms in the complexes.

As far as the dissociation of these complexes is concerned, care must be taken in choosing balanced values for the energies of the fragments for the possible dissociation channels.

For a positron-diatomic molecule complex $e^{+} \mathrm{MX}$, where $\mathrm{M}=\mathrm{Li}$ or $\mathrm{Be}$ and $\mathrm{X}=\mathrm{O}$ or $\mathrm{F}$, the possible fragmentations are $e^{+} \mathrm{M}+\mathrm{X}, \mathrm{M}^{+}+\mathrm{PsX}, \mathrm{M}+e^{+} \mathrm{X}$, and $\mathrm{PsM}+\mathrm{X}^{+} . \mathrm{Al}-$ though not all the energy values of the fragments are known, one can safely assume that the $\mathrm{PsM}+\mathrm{X}^{+}$dissociation pattern has the highest energy with respect to the other possibilities. This is due first to the large ionization potential of $\mathrm{X}(0.5005$ hartree for $\mathrm{O}$ and 0.6403 hartree for $\mathrm{F}),{ }^{35}$ at least twice as large as the positronium (Ps) ground state energy $(-0.25$ hartree); second, to the usually small binding energy of Ps to metal atoms (for instance, the binding energy of Ps to $\mathrm{Li}$ in the PsLi complex is just 0.012084 hartree). ${ }^{8,34}$ Moreover, we believe it is reasonable to also discard the $\mathrm{M}+e^{+} \mathrm{X}$ channel, since the possibility of obtaining binding between $e^{+}$and $\mathrm{X}$ is hindered by the small polarizability of X.

To support this conclusion, we stress the fact that even for $\mathrm{HF}$ and $\mathrm{H}_{2} \mathrm{O}$, both polar molecules, DMC did not show binding with the positron. ${ }^{12}$ Although this is not a proof, it strongly suggests that $e^{+} \mathrm{O}$ and $e^{+} \mathrm{F}$ probably are not bound.

Accepting these conclusions, we are left only with $e^{+} \mathrm{M}+\mathrm{X}$ and $\mathrm{M}^{+}+\mathrm{Ps} \mathrm{X}$ as possible fragmentations. To compute the total energy for both channels we use the ECG results for $e^{+} \mathrm{Li}, e^{+} \mathrm{Be}, \mathrm{Li}^{+}$, and $\mathrm{Be},{ }^{8,34}$ supplemented by the FN-DMC results for $\mathrm{O}, \mathrm{F}^{36} \mathrm{PsO}$, and $\mathrm{PsF} .{ }^{11}$ The energy for these systems is shown in Table III. Moreover, we estimate the $\mathrm{Be}^{+}$energy $(-14.3248$ hartree) subtracting the ionization potential $(0.3426 \text { hartree })^{35}$ from the total energy of Be.

Using these results, we end up with an energy of $-107.2499(3)$ hartree for $e^{+} \mathrm{Li}$ and $\mathrm{F}$, and an energy of $-107.3518(8)$ hartree for $\mathrm{Li}^{+}$and PsF. This last fragmentation, similar to the one found for $e^{+} \mathrm{LiH}$ (i.e., $\mathrm{Li}^{+}$and $\mathrm{PsH}$ ), is primarily driven by the small value of the $\mathrm{Li}$ ionization potential. Stated differently, for $e^{+} \mathrm{BeO}$ we obtain -89.6425(5) hartree for $\mathrm{Be}^{+}$and $\mathrm{PsO}$, and -89.7208(4) hartree for $e^{+} \mathrm{Be}$ and $\mathrm{O}$, so that the most stable dissociation fragments present a positron bound to an atom.

Using the lowest energy dissociation threshold for the two systems one gets a DE of $0.077(1)$ hartree for $e^{+} \mathrm{LiF}$, and 0.090(2) hartree for $e^{+} \mathrm{BeO}$. Both these values are larger than the APA, and this fact means that the two positronmolecule complexes do not dissociate after positron addition to the parent molecules. This outcome is different from what we found for the addition of $e^{+}$to $\mathrm{LiH}$, where the $e^{+} \mathrm{LiH}$ complex breaks up due to the excess of the APA with respect to the DE. ${ }^{21}$ Therefore, it does not appear necessary for LiF and $\mathrm{BeO}$ to use a third body, and a simple positron addition will give birth to metastable complexes in rotovibrational excited states.

As previously stated, the possibility to produce these stable species could give the chance to experimentally detect stable positron complexes. Roughly speaking, a mean annihilation lifetime on the order of $10^{-9} \mathrm{~s}$ is expected for these systems, and this may be large enough to allow a spectroscopical analysis in the reaction chamber by means of Fourier transform infrared spectroscopy. This outcome requests both a sufficient concentration of $e^{+} \mathrm{MX}$, and a frequency shift, with respect to the parent molecule, large enough that the vibrational spectrum of the complex does not overlap with the neutral molecule one. Unfortunately, the large uncertainty in $\omega_{e}$ does not allow us a quantitative prediction of this frequency shift. Moreover, before we attempt such an experiment an estimate of the lifetime for the metastable states of $e^{+} \mathrm{MX}$ must be given.

Conversely, one could exploit these results by looking for vibrational resonances during the positron-molecule collisions in the energy range $[0, \mathrm{DE}-\mathrm{APA}]$ of the incoming positron. In this range, the two molecules cannot dissociate and there are chances for the positron to be trapped in a Feshbach resonance due to the existence of stable excited vibrational states of the $e^{+} \mathrm{MX}$ systems. Although this experiment appears feasible, it requests low-energy positron sources with a high degree of monochromaticity, and an accurate theoretical prediction of the resonance positions requires the development of new tools to deal with the scattering of positrons on complicated targets. Such a development is under way in our laboratory. ${ }^{37}$

Moreover, positrons having kinetic energy larger than the difference DE-APA can open the various fragmentation channels depending on the excess of their relative energies. For instance, the collision between positron and $\mathrm{BeO}$ can produce $e^{+} \mathrm{Be}$ and $\mathrm{O}$ as fragments, so that the annihilation of $e^{+}$with the electronic cloud of Be can be directly recorded from the $2 \gamma$ photons. Moreover, it might be possible to detect the stable state of PsF, a system that, differently from $\mathrm{PsCl}$ and $\mathrm{PsBr}$, has not been prepared in solution. ${ }^{3}$

In conclusion, we have presented accurate APA and DE for $e^{+} \mathrm{LiF}$ and $e^{+} \mathrm{BeO}$ systems computed by means of $\mathrm{FN}-$ DMC. These results allow us to discuss possible mechanisms of formation for metastable positron-molecule complexes by direct attachment of $e^{+}$to the molecules, and the possibility to produce $e^{+} \mathrm{M}$ and PsX systems. It would be now interesting to compute the $\Gamma_{2 \gamma}$ annihilation rate for these complexes, in order to predict their mean lifetime after $e^{+}$addition. Unfortunately, more technical work on the method appears to be necessary before these calculations can be carried out for these large systems. 
The authors would like to thank J. Mitroy and G. Gribakin for many useful comments and suggestions, and Rocco Martinazzo for clarifying discussion on resonances. Financial support by the Universita' degli Studi di Milano is gratefully acknowledged. The authors are indebted to the Centro CNR per lo Studio delle Relazioni tra Struttura e Reattivita' Chimica for grants of computer time.

${ }^{1}$ M. H. Weber, S. Tang, S. Berko, B. L. Brown, K. F. Canter, K. G. Lynn, A. P. Mills, Jr., L. O. Roellig, and A. J. Viescas, Phys. Rev. Lett. 61, 2542 (1988).

${ }^{2}$ G. Consolati, R. Rurali, and M. Stefanetti, Chem. Phys. 273, 493 (1998).

${ }^{3}$ P. Castellaz, J. Major, C. Mujica, H. Schneider, A. Seeger, A. Siegle, H. Stroll, and I. Billard, J. Radioanal. Nucl. Chem. 210, 457 (1996).

${ }^{4}$ K. Iwata, G. F. Gribakin, R. G. Greaves, C. Kurz, and C. M. Surko, Phys. Rev. A 61, 022719 (2000).

${ }^{5}$ D. M. Schrader, F. M. Jacobsen, N. Frandsen, and U. Mikkelsen, Phys. Rev. Lett. 69, 57 (1992).

${ }^{6}$ K. Strasburger, Chem. Phys. Lett. 253, 49 (1996).

${ }^{7}$ R. M. Nieminen, E. Boronski, and L. Lantto, Phys. Rev. B 32, 1377 (1985).

${ }^{8}$ G. G. Ryzhikh, J. Mitroy, and K. Varga, J. Phys. B 31, 3965 (1998).

${ }^{9}$ K. Strasburger, J. Chem. Phys. 111, 10555 (1999).

${ }^{10}$ D. Bressanini, M. Mella, and G. Morosi, Phys. Rev. A 57, 1678 (1998).

${ }^{11}$ D. Bressanini, M. Mella, and G. Morosi, J. Chem. Phys. 108, 4756 (1998).

${ }^{12}$ D. Bressanini, M. Mella, and G. Morosi, J. Chem. Phys. 109, 1716 (1998).

${ }^{13}$ D. Bressanini, M. Mella, and G. Morosi, J. Chem. Phys. 109, 5931 (1998).

${ }^{14}$ M. Mella, G. Morosi, and D. Bressanini, J. Chem. Phys. 111, 108 (1999).

${ }^{15}$ M. Mella, G. Morosi, and D. Bressanini, J. Chem. Phys. 112, 1063 (2000).

${ }^{16}$ B. L. Hammond, W. A. Lester, Jr., and P. J. Reynolds, Monte Carlo
Methods in Ab Initio Quantum Chemistry, 1st ed. (World Scientific, Singapore, 1994)

${ }^{17}$ T. Yoshida, G. Miyako, N. Jiang, and D. M. Schrader, Phys. Rev. A 54, 964 (1996).

${ }^{18}$ T. Yoshida and G. Miyako, Phys. Rev. A 54, 4571 (1996).

${ }^{19}$ N. Jiang and D. M. Schrader, J. Chem. Phys. 109, 9430 (1998); Phys. Rev. Lett. 81, 5113 (1998).

${ }^{20}$ G. Morosi, M. Mella, and D. Bressanini, J. Chem. Phys. 111, 6755 (1999).

${ }^{21}$ M. Mella, G. Morosi, D. Bressanini, and S. Elli, J. Chem. Phys. 113, 6154 (2000).

${ }^{22}$ J. Mitroy and G. G. Ryzhikh, J. Phys. B 33, 3495 (2000).

${ }^{23}$ K. E. Schmidt and J. W. Moskowitz, J. Chem. Phys. 93, 4172 (1990).

${ }^{24}$ J. W. Moskowitz and K. E. Schmidt, J. Chem. Phys. 97, 3382 (1992).

${ }^{25}$ D. Bressanini, M. Mella, and G. Morosi, Chem. Phys. Lett. 272, 370 (1997).

${ }^{26}$ D. Bressanini, M. Mella, and G. Morosi, Phys. Rev. A 55, 200 (1997).

${ }^{27}$ K. P. Huber and G. Herzberg, Molecular Spectra and Molecular Structure. Constants of Diatomic Molecules (Van Nostrand Reinhold, New York, 1979).

${ }^{28}$ G. L. Gutsev, M. Nooijen, and R. J. Bartlett, Chem. Phys. Lett. 276, 13 (1997).

${ }^{29}$ M. W. J. Bromley and J. Mitroy, J. Phys. B 33, L325 (1999).

${ }^{30}$ J. Mitroy and G. G. Ryzhikh, J. Phys. B 32, 1375 (1999).

${ }^{31}$ M. W. J. Bromley, J. Mitroy, and G. G. Ryzhikh, Nucl. Instrum. Methods 171, 47 (2000).

${ }^{32}$ G. G. Ryzhikh and J. Mitroy, J. Phys. B 31, 4459 (1998).

${ }^{33}$ G. G. Ryzhikh and J. Mitroy, J. Phys. B 31, 5013 (1998).

${ }^{34}$ J. Mitroy and G. G. Ryzhikh, J. Phys. B (submitted).

${ }^{35}$ C. E. Moore, NIST Report No. NSRDS-NBS34, 1970.

${ }^{36}$ A. Lüchow and J. B. Anderson, J. Chem. Phys. 105, 7573 (1996).

${ }^{37}$ S. Chiesa, M. Mella, D. Bressanini, and G. Morosi (unpublished). 